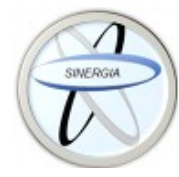

\title{
Análisis clínico y patológico del coriocarcinoma gestacional
}

\author{
Clinical and pathological analysis of gestational
}

\author{
${ }^{1}$ Dra. Irene Brenes Valverde \\ Hospital Nacional Psiquiátrico, San José, Costa Rica \\ (iD https://orcid.org/0000-0002-1938-7976 \\ ${ }^{2}$ Dra. Arianne Mora Morales \\ Hospital Nacional Psiquiátrico, San José, Costa Rica \\ (iD https://orcid.org/0000-0001-5494-6086 \\ ${ }^{3}$ Dra. Karen Prendas Navarro \\ Investigadora independiente, Cartago, Costa Rica \\ (iD) https://orcid.org/0000-0002-4675-8779
}

Recibido

$08 / 12 / 2021$
Corregido

$15 / 01 / 2022$
Aceptado

$10 / 02 / 2022$

\section{RESUMEN}

El coriocarcinoma es una patología infrecuente, pero potencialmente fatal si no se trata. Está incluida dentro de las Neoplasias Trofoblásticas Gestacionales (NTG), un grupo de tumores malignos altamente invasivos, metastásicos y vascularizados. Su presentación tras una gestación a término conlleva peor pronóstico que tras un aborto o una mola hidatiforme, porque refleja un retraso en el diagnóstico y tratamiento. Los elevados niveles de Gonadotropina Coriónica Humana beta ( $\beta$-hCGesenciales su diagnóstico. El tratamiento debe de ser precoz, basado en quimioterapia con múltiples fármacos idealmente, aunque en ocasiones también se incluyen la cirugía y radioterapia.

PALABRAS CLAVE: gestacional; gonadotropina coriónica humana; metástasis

\section{ABSTRACT}

Choriocarcinoma is a rare but potentially fatal condition if not treated. It is included within the gestational trophoblastic neoplasms (GTN), a group of highly invasive, metastatic and highly vascular malignant tumors. Its presentation after a full-term pregnancy carries a worse prognosis than after an abortion or a hydatidiform mole because it reflects a delay in diagnosis and treatment. Elevated beta Human Chorionic Gonadotropin ( $\beta$-hCG) levels, Doppler ultrasound, imaging tests, and clinical manifestations are essential to diagnose postpartum choriocarcinoma. Treatment must be early, based on polychemotherapy, although sometimes surgery and radiotherapy are also included. 
KEYWORDS: gestational; human chorionic gonadotropin; metastasis

${ }^{1}$ Médica general, graduada de la Universidad Latina de Costa Rica (U. Latina). Cód. MED16924. Correo: dra.brenesv@gmail.com

${ }^{2}$ Médica general, graduada de la Universidad Autónoma de Centroamérica (UACA). Cód.MED16277. Correo: ariannemora@gmail.com

${ }^{3}$ Médica general, graduada de la Universidad de Ciencias Médicas (UCIMED). Cód. MED16605 . Correo: naty.p.n95@gmail.com

\section{INTRODUCCIÓN}

La enfermedad trofoblástica gestacional (ETG) agrupa diferentes patologías proliferativas del trofoblasto placentario. Estas manifestaciones se caracterizan por una hipersecreción de $\beta$-hCG. Alrededor de los cinco a seis días de embarazo, el cigoto se convierte en blastocisto y este forma una capa interna de células aplanadas, el citotrofoblasto y otra externa que es el sincitotrofoblasto, encargado de invadir el endometrio y la vasculatura uterina. Ambas capas son el origen de la placenta (20). Estos trastornos proliferativos dan lugar a patologías no neoplásicas como la mola hidatiforme (parcial o completa) y neoplásicas como el tumor epiteloide trofoblástico (ETT), tumor trofoblástico del sitio placentario (PSTT) y coriocarcinoma, del cual se enfatizará en esta revisión de tema.

La mola hidatiforme se caracteriza por un crecimiento anormal del trofoblasto como resultado de una fertilización anormal. Las vellosidades coriónicas se edematizan y forman cúmulos de vesículas y adquieren un aspecto macroscópico de "racimos de uvas" .La mola hidatiforme se puede subdividir en dos tipos: completa y parcial. Las completas no contienen partes fetales, las vellosidades coriónicas son anómalas, y todo el contenido genético es paterno, aportado por dos espermatozoides o uno diploide (46 XX o 46 $X Y)$. La mola parcial puede contener partes fetales y algunas vellosidades coriónicas normales, y casi siempre muestra triploidía, ya que el óvulo es fecundado por dos espermatozoides (o uno diploide) (21).
La manifestación clínica más frecuente es el sangrado transvaginal, sin embargo, el coriocarcinoma no posee síntomas o signos específicos, por lo que dificulta su diagnóstico.

De forma general, el diagnóstico precoz del coriocarcinoma es importante, ya que contribuye a una posible terapia curativa cuando se utilizan regímenes de quimioterapia adecuados en caso de captación temprana.

El objetivo de esta revisión de tema es conocer las características clínicas y anatomopatológicas del coriocarcinoma gestacional para un manejo oportuno, pues la característica principal de esta patología es que conforma la forma histológica más agresiva de todos los tipos de ETG, caracterizado por diseminación metastásica temprana si no se detecta de forma precoz (1).

\section{MÉTODO}

En el presente artículo realizamos una revisión de tema mediante exhaustivas búsquedas bibliográficas donde al final se utilizaron 22 referencias de literatura científica tanto en el idioma inglés como español, actualizada de los últimos 5 años sobre la Enfermedad Trofoblástica Gestacional enfocándonos de forma detallada en el Coriocarcinoma, con el propósito de comparar información de diferentes fuentes, analizar y sintetizar estudios, para así actualizarnos sobre el tema y trasmitir nuevos conocimientos de manera básica, clara y sencilla al lector. Durante la búsqueda, se utilizaron palabras 
claves como "neoplasia", "quimioterapia", "estadificación", "gonadotropina coriónica humana", y "metástasis". Se consultaron bases de datos como Google Scholar, Medscape, Sientific Electronic Library Online (SciELO), y PubMed.

\section{DEFINICIÓN}

El Coriocarcinoma gestacional es un tumor de carácter maligno, agresivo, desarrollado a partir de la proliferación del citotrofoblasto y sincitotrofoblasto velloso que sufre hiperplasia y anaplasia, con invasión del miometrio, vasos sanguíneos y metástasis a distancia. Típicamente ocurre con mayor frecuencia después de un embarazo molar completo (22).

Es una neoplasia sumamente vascular que se caracteriza por necrosis y ausencia de vellosidades coriónicas, al contrario que en la mola hidatiforme. Dentro del útero, el coriocarcinoma invade la rica vascularización de la decidua, que lo caracteriza por su gran capacidad invasiva vascular para diseminarse por todo el cuerpo. Es un tumor que produce altos niveles de factores de crecimiento angiogénicos y son capaces de remodelar la vasculatura uterina, lo cual puede provocar hemorragia, clínicamente visible como primera manifestación clínica. Aproximadamente el $30 \%$ de los casos tienen enfermedad metastásica al momento del diagnóstico.

\section{ANATOMÍA PATOLÓGICA}

- Macroscópicamente: el coriocarcinoma se observa como una masa uterina irregular, única o múltiple, con características necróticas y hemorrágicas, las cuales tienden a ser extensas y podrían llegar a ocupar la totalidad del tumor (21). El crecimiento es difuso e infiltrativo, con nódulos de color violáceo a rojo oscuro en su superficie. La lesión primaria puede encontrarse principalmente en útero, como también en trompas uterinas, ovarios, cuello uterino, o sitios afectados por un embarazo ectópico.

- Microscópicamente: se caracteriza por núcleos de citotrofoblasto mononucleares rodeados por bordes de sincitotrofoblasto multinucleados. Las células tumorales se encuentran principalmente en la periferia de la lesión con hemorragia y necrosis en el centro. Además, hay presencia de pleomorfismo citológico marcado, agrandamiento nuclear y actividad mitótica importante. Para el diagnóstico histopatológico, es requisito la ausencia de las vellosidades coriónicas.

\section{EPIDEMIOLOGÍA}

Se estima que el coriocarcinoma corresponde entre 2 a $5 \%$ de todos los casos de NTG. La incidencia es relativamente baja en Norte América y Europa siendo estimada 1 en 40.000 embarazos y en 1 en 40 casos de embarazo molar; en cuanto a lo reportado en Suroeste Asiático, la incidencia es de 9.2 en 40000 y en Japón de 3.3 por cada 40.000 embarazos $(10,15)$.

En el caso específico de la mola hidatiforme, se considera que el $80 \%$ es benigna e involuciona espontáneamente; del 15 a 20\% es persistente y del 2 al $3 \%$ se transformará en coriocarcinoma (10). Según Shanbhogue, Lalwani y Menias, aproximadamente el 50\% de los casos surgen de embarazo molar, $25 \%$ de aborto involuntario, embarazo ectópico y, finalmente, un $25 \%$ corresponde a embarazo de término o pretérmino (13).

FACTORES DE RIESGO 
Dentro de los factores de riesgo relacionados con el coriocarcinoma se pueden describir los siguientes $(2,13)$ :

- Edad materna menor a 20 años y mayor a 40 años.

- Historia previa de enfermedad trofoblástica.

- Multiparidad en mujeres con más de 5 gestas previas.

- Edad paterna mayor a 40 años (aún en estudio).

- Antecedente de haber utilizado anticonceptivos orales en un tiempo mayor a 7 años (riesgo 2 a $6.4 \%$ mayor).

- Tabaquismo.

- Exposición a pesticidas o herbicidas.

- Tipo de sangre materna A o AB.

\section{PRESENTACIÓN CLÍNICA}

Las manifestaciones clínicas se relacionan con la localización y la presencia o no de metástasis, debido a que posterior a una gestación no molar los síntomas y signos se pueden mostrar de forma sutil (10).

La manifestación más común es el sangrado vaginal persistente e irregular a las seis semanas postparto en un $74 \%$ de las mujeres con coriocarcinoma, de forma escasa o abundante, incluso pudiendo llegar a complicarse en un shock hemorrágico (2). Alrededor de un $50 \%$ tendrán una altura uterina mayor a la esperada para la edad gestacional y $30 \%$ presentarán aumento del tamaño en uno o ambos ovarios (13). Otros síntomas asociados y menos específicos son vómitos, dolor abdominal, síntomas relacionados con la preeclampsia, hipermemésis gravídica, anemia o hipertiroidismo (14).

De forma general, dentro de las NTG, se ha encontrado una incidencia de metástasis del $19 \%$, siendo el coriocarcinoma a quien más se le relaciona. La ruta de diseminación más común es la hematógena, y los lugares más frecuentes de metástasis son: pulmón (60$95 \%)$, vagina $(40-50 \%)$, vulva o cérvix (10$15 \%)$, cerebro (5-15\%), hígado (5-15\%), ovarios, trompas uterinas, riñón e incluso se han reportado casos de metástasis a coroides $(0.2 \%)(10,12)$.

Algunos de los síntomas que se han documentado relacionado con la metástasis son: cefalea, alteración del estado de conciencia, convulsiones, vértigo, alteraciones de la visión, disnea, hemoptisis, dolor torácico, dolor abdominal o hematuria, siendo los síntomas respiratorios los más frecuentes por ser el sitio de metástasis de mayor frecuencia $(2,10,12,14)$.

\section{DIAGNÓSTICO}

Debido a que el coriocarcinoma no posee síntomas o signos específicos, su diagnóstico es difícil y en muchas ocasiones tardío, afectando el pronóstico de la paciente (3).

Se debe realizar historia clínica, incluyendo fecha, duración y evolución del embarazo actual y los previos. El examen físico debe ser completo, tomando en cuenta la búsqueda de signos o síntomas que sugieran metástasis. Se debe incluir especuloscopía, por la gran frecuencia de diseminación a vagina y la palpación bimanual debe realizarse en busca de masas anexiales y características del útero.: En cuanto al $\beta$-hCG, el coriocarcionoma puede presentar un retraso en el diagnóstico tras una gestación no molar, ya que no se miden niveles de $\beta$-hCG ni estudio histológico de la placenta de forma rutinaria, por lo que se debe considerar en estos casos que las pacientes con valores crecientes de $\beta$-hCG tienen coriocarcinoma hasta que se demuestre lo contrario (8).

En caso de embarazo molar previo, la medición semanal de $\beta$-hCG es parte del 
seguimiento en estas pacientes, por lo que una persistencia de niveles altos de esta hormona, nos puede hacer sospechar del desarrollo de una NTG. El diagnóstico se basa en los criterios de la Federación Internacional de Ginecología y Obstetricia (FIGO) los cuales son $(5,9,10)$ :

- Los niveles semanales de $\beta$-hCG se estabilizan permaneciendo dentro del $10 \%$ del resultado anterior durante tres semanas.

- El nivel de $\beta$-hCG aumenta más de $10 \%$ en tres valores registrados durante dos semanas.

- Persistencia de $\beta$-hCG sérica detectable por más de seis meses después de la evacuación molar.

\section{Biopsia}

Este método se ha convertido en una herramienta útil en el diagnóstico, vigilancia y detección de recurrencias en caso de resistencia a fármacos, sin embargo, actualmente no se aconseja una biopsia tumoral de la lesión si no existe garantía de controlar el sangrado, ya que son lesiones con un gran componente necrótico y hemorrágico. Por lo tanto, la presentación clínica de una NTG es más importante para determinar e iniciar el tratamiento que para un diagnóstico histológico preciso $(2,3,6)$.

\section{Estudios de imagen}

- La ecografía, en combinación con la historia clínica y los niveles de $\beta$-hCG, es un método diagnóstico de primera línea para la NTG. Se puede realizar tanto transabdominal como transvaginal. El coriocarcinoma se observa como una masa que agranda el tamaño del útero. Su heterogeneidad se relaciona con las áreas de necrosis y hemorragia. El tumor es hipervascular al Doppler y puede extenderse a parametrio $(1,2)$.

- La radiografía de tórax, es útil para evaluar metástasis pulmonar, siendo este el principal lugar de diseminación. Los hallazgos los podemos observar como densidades redondeadas, con un patrón alveolar o en "tormenta de nieve", asociado también a derrame pleural o patrón embólico causado por oclusión de la arterial pulmonar.

- La tomografía computarizada tóracoabdómino-pélvica puede revelar micrometástasis pulmonar no detectadas en la radiografía de tórax hasta en un $40 \%$ de las NTG (2) y para las pacientes en cuyos casos los tumores no pueden detectarse mediante la ecografía, la resonancia magnética es útil para su localización y además para descartar metástasis a sistema nervioso central $(1,2)$.

\section{ESTADIFICACIÓN}

La NTG se clasifica usando una combinación del Sistema de Estadificación de la Federación Internacional de Ginecología y Obstetricia (FIGO) del 2002 y el Sistema de Calificación de Pronóstico de la Organización Mundial de la Salud (OMS). Los factores de riesgo que se describen a continuación (Tabla 1) predicen la resistencia a la quimioterapia con un solo fármaco, ya sea Metrotrexato o Actinomicina D $(5,10,11)$ :

- Estadío I: Tumor limitado al cuerpo del útero

- Estadío II: Tumor que se extiende a los anexos o vagina, pero limitado a estructuras genitales. 
- Estadío III: Metástasis pulmonares visibles en radiografía de tórax, con o sin compromiso uterino, pélvico o vaginal.

- Estadío IV: Enfermedad metastásica a distancia, fuera de los pulmones, pelvis o vagina.

Posteriormente, se asigna la puntuación según los factores de riesgo propuestos por la OMS, la cual va especificada seguida del número de estadiaje (Ej: II:2).

\section{TRATAMIENTO}

Las opciones de tratamiento para la NTG difieren según el tipo y la etapa de la enfermedad, esto incluye la quimioterapia, dilatación y legrado, histerectomía o una combinación de estos (7). En el caso del coriocarcinoma siempre está indicada la quimioterapia, la cual debe iniciarse de forma oportuna para evitar complicaciones hemorrágicas y diseminación metastásica (2).

\section{Quimioterapia:}

Cuando se trata de una enfermedad de alto riesgo, estadio IV o estadio II-III con puntuación igual o superior a 7 , el tratamiento se basa en esquemas de poliquimioterapia y suele ser prolongada. EI esquema EMA-CO puede ofrecer tasas de curación entre el 54-90\%. Este consiste en Etopósido, Metotrexato, Dactinomicina, Ciclofosfamida y Vincristina. Debe continuarse hasta que la $\beta$-hCG sea indetectable y por 3 semanas más. Finalmente, se administra al menos 3 ciclos más como consolidación $(1,2,4,5)$. En caso de resistencia a la quimioterapia (un tercio de las pacientes de alto riesgo), el régimen debe modificarse y es necesario introducir algún otro método de tratamiento, ya sea resección de la metástasis o radioterapia $(5,7)$.

Posterior a la quimioterapia es posible que queden lesiones residuales en áreas metastásicas o en el útero. En este caso no es necesario extirparlas, ya que no reduce el riesgo de recurrencia $(2,5,7)$.

\section{Manejo quirúrgico:}

La histerectomía en pacientes de bajo riesgo puede disminuir el tiempo de la quimioterapia, especialmente en mujeres con paridad satisfecha. En las pacientes de alto riesgo y con metástasis, la histerectomía no mejora el pronóstico, por lo que no se recomienda (2). En ambos casos, la cirugía puede provocar hemorragias masivas del tumor altamente vascularizado (5).

El legrado uterino tiene un papel limitado en la evacuación de la NTG. Se ha reservado para pacientes con hemorragia posparto o postaborto y un nivel elevado de $\beta$-hCG para determinar si el diagnóstico es NTG o productos retenidos de la concepción.

Podemos decir que la paciente entra en remisión cuando los niveles de $\beta$-hCG se tornan indetectables por tres semanas consecutivas y se define persistencia de la enfermedad si estos niveles se mantienen o incrementan en dos mediciones consecutivas en un intervalo de dos semanas. En estos casos, la cirugía podría ser una opción con fines curativos.

\section{SEGUIMIENTO}

El seguimiento de la NTG es de suma importancia. Durante su tratamiento, se deben realizar mediciones de $\beta$-hCG cada semana hasta obtener 3 mediciones semanales consecutivas normales. Posteriormente cada mes por un año (en pacientes de alto riesgo por 18 meses), y luego medir cada 6 meses hasta por 5 años. 
En algunas pacientes se sugiere incluso la medición del nivel de $\beta$-hCG cada 6 meses hasta el final de la vida. Estas pacientes deben utilizar medidas anticonceptivas eficaces y es importante considerar el asesoramiento psicológico $(5,10,16)$.

\section{PRONÓSTICO Y SUPERVIVENCIA}

El pronóstico se puede clasificar según el subtipo histológico, extensión y duración de la enfermedad, niveles de $\beta$-hCG y la extensión del tratamiento previo (17). En estos casos la estadificación FIGO es el principal factor que define el pronóstico.

En estadío I, las pacientes suelen tener un pronóstico favorable después de la histerectomía total. La tasa de supervivencia a 10 años puede llegar al 100\%. En estadío II-III sin presencia de metástasis, al desarrollar resistencia al tratamiento y al agregar un tratamiento de segunda línea, pueden tener una remisión hasta en el $85 \%$ de los casos $(1,19)$. Sin embargo, estos estadíos con presencia de metástasis o en estadio IV, el pronóstico es relativamente malo y según las estadísticas de Gran Bretaña, la tasa de supervivencia a 10 años es de alrededor del 49\% (1).

Cabe resaltar que después de introducir la quimioterapia combinada como tratamiento primario en este grupo, la tasa de supervivencia mejoró drásticamente, logrando una remisión de hasta el $60-70 \%$ de los casos $(1,17,18)$.

\section{CONCLUSIONES}

El coriocarcinoma es una enfermedad poco frecuente pero que puede poner en peligro la vida de la paciente a corto plazo, sobre todo en el caso de existir metástasis, las cuales son muy frecuentes en esta tumoración, dada su diseminación vía hemática.
Se destaca la importancia de la exploración ecográfica y la determinación de $\beta$-hCG, herramientas imprescindibles para el diagnóstico y seguimiento. Es importante el tratamiento precoz, dada la rapidez con la que se puede desarrollar y extender este tumor (3). Las pacientes con un diagnóstico con sospecha de NTG o confirmado de coriocarcinoma, deben ser remitidas urgentemente a un centro especializado para una evaluación adicional que incluya estudios de imagen y descartar la presencia de enfermedad metastásica (6).

El tratamiento de elección en el coriocarcinoma es la quimioterapia, ya que se puede usar con intención curativa aún en el contexto de una enfermedad avanzada, sin embargo, puede ser necesario un tratamiento quirúrgico, especialmente en los casos de complicaciones potencialmente mortales, relacionadas con mayor frecuencia con una hemorragia masiva del tumor. Se sugiere el esquema EMA-CO para pacientes de alto riesgo $(3,5)$.

Todas las pacientes con NTG deben ser monitoreadas con mediciones seriadas de $\beta$ hCG sérica al inicio del tratamiento y luego a intervalos según el riesgo, ya que la persistencia en estos niveles sugiere recurrencia de la enfermedad, siendo un factor pronóstico entre estos pacientes.

\section{Los autores declaran no tener conflicto de interés.}

\section{REFERENCIAS}

1. Xuan Feng, Zhi Wei, Sai Zhang, Yan Du and Hongbo Zhao. (2019). A Review on the Pathogenesis and Clinical Management of Placental Site Trophoblastic Tumors. Frontiers in Oncology. Vol 9, Article 937. doi: 10.3389/fonc.2019.00937

2. Andrea Agustín O., Ricardo Savirón C., Diego Lerma P., Ana Herrero I., José Manuel Campillos M. (2015). Casos Clínicos. Coriocarcinoma 
Postgestacional. Revista Chilena de Obstetricia y Ginecologia. 80(5): 405 - 411

3. Celia Aparicio-Rubio, Eva Hernández-Lorente, Corina Escoin-Pérez. (2017). Coriocarcinoma: A propósito de un caso. Farmacia Hospitalaria. I Vol. 41 | No 6 | 692 - 693 |

4. MsC. Vicia Sánchez Abalos, MsC. Carmen Bosch Costafreda y MsC. Tamara Mercedes Sánchez Abalos. (2014). Coriocarcinoma con metástasis pulmonar. MEDISAN. 18(5):705

5. Patrycja Wreczycka-Cegielny, Tomasz Cegielny, Marcin Oplawski, Wlodzimierz Sawicki, Zbigniew Kojs. (2018). Current treatment options for advanced choriocarcinoma on the basis of own case and review of the literature. The Chair and Clinic of Obstetrics, Gynaecologic Diseases and Oncological Gynaecology of the Second Faculty of Medicine of Warsaw Medical University, Poland. vol. 89, no. 12, 711-715. DOI: 10.5603/GP.a2018.0120

6. Jiao, L., Ghorani, E., Sebire, N. and Seckl, M., 2016. Intraplacental choriocarcinoma: Systematic review and management guidance. Gynecologic Oncology, 141(3), pp.624-631.

7. Fen Ning, Houmei Hou, Abraham N. Morse, Gendie E. Lash. (2019). Understanding and management of gestational trophoblastic disease. F1000Research. 8(F1000 Faculty Rev):428 (https://doi.org/10.12688/f1000research.14953.1)

8. Mishu Mangla, Deepak Singla, Harpreet Kaur, Sushmita Sharma. (2017). Unusual clinical presentations of choriocarcinoma: A systematic review of case reports. Taiwanese Journal of Obstetrics \& Gynecology. 56 (1-8).

9. 9. Kong $Y$, Yang J, Jiang F, Zhao J, Ren T, Li J, Wang X, Feng F, Wan X, Xiang Y. (2017). Clinical characteristics and prognosis of ultra high-risk gestational trophoblastic neoplasia patients: A retrospective cohort study. PubMed. Gynecol Oncol;146(1):81. Epub 2017 Apr 29.

10. Ngan HYS, Seckl MJ, Berkowitz RS, Xiang Y, Golfier F, Sekharan PK, Lurain JR, Massuger L. (2018). Update on the diagnosis and management of gestational trophoblastic disease. PubMed. Int J Gynaecol Obstet. 2018;143 Suppl 2:79.

11. Gestational Trophoblastic Neoplasms (Female Reproductive Organs). In: AJCC Cancer Staging Manual, Eighth Edition, Amin MB (Ed), Springer, Chicago 2017.

12. A.Cumminga, J.Balanzario-Cuevasa, J.VitarSandovala, J.Monjaras-Guerraa, J.RodríguezRoblesa, I.Cortés-Tapiab, G.Regalado-Porrasc, E.Blancasd, V.E.Corona-Montes. (Setiembres 2016). Coriocarcinoma testicular con metástasisa coroides. Revista Mexicana de Urología, vol. 76, páginas 387-391.

13. Shanbhogue, A. K., Lalwani, N., \& Menias, C. O. (2013). Gestational Trophoblastic Disease. Radiologic Clinics, 51(6), 1023-1034.

14. Dhanda, S., Ramani, S. and Thakur, M., 2014. Gestational Trophoblastic Disease: A Multimodality Imaging Approach with Impact on Diagnosis and Management. Radiology Research and Practice, 2014, pp.1-12.

15. Wreczycka-Cegielny, P., Cegielny, T., Oplawski, M., Sawicki, W. and Kojs, Z., 2018. Current treatment options for advanced choriocarcinoma on the basis of own case and review of the literature. Ginekologia Polska, 89(12), pp.711715.

16. Santaballa A, García $Y$, Herrero A, Laínez N, Fuentes J, De Juan A, Rodriguez Freixinós $V$, Aparicio J, Casado A, García-Martinez E. SEOM clinical guidelines in gestational trophoblastic disease (2017). Clinical and Translational Oncology. 2017.

17. Brown Jubilee, Naumann R. Wendel, Seckl Michael J, Schink Julian. 15 years of progress in gestational trophoblastic disease: Scoring, standardization, and salvage. Gynecologic Oncology, $\quad$ ELSEVIER. 2016. www.elsevier.com/locate/ygyno

18. Freitas F, Braga A, Viggiano M, et al. Gestational trophoblastic neoplasia lethality among Brazilian women: A retrospective national cohort study. Gynecol Oncol 2020; 158:452.

19. Balachandran K, Salawu A, Ghorani E, et al. When to stop human chorionic gonadotrophin (hCG) surveillance after treatment with chemotherapy for gestational trophoblastic neoplasia (GTN): A national analysis on over 4,000 patients. Gynecol Oncol 2019; 155:8.

20. Jean-Jacques Candelier (2016) The hydatidiform mole, Cell Adhesion \& Migration, 10:1-2, 226235, DOI: 10.1080/19336918.2015.1093275

21. Robbins LS, Cotran SR, Kumar V. Patología Estructural y Funcional. 10ma ed. España: Elsevier 2018.

22. Ma, N., Litkouhi, B., \& Mannion, C. M. (2016). FIGO Stage III Metastatic Gestational Choriocarcinoma Developed From an Antecedent Partial Hydatidiform Molar Pregnancy Bearing a Numerical Chromosomal Aberration 68, XX: A Case Report and Literature Review. International journal of gynecological pathology : official journal of the International Society of Gynecological Pathologists, 35(2), 162-166. https://doi.org/10.1097/PGP.0000000000000215 